\title{
Home damage estimation after disasters using crowdsourcing ideas and Convolutional Neural Networks
}

\author{
Zhanghua $\mathrm{Li}^{1,}$,, Kun Tian ${ }^{2, b}$ Fushan Wang ${ }^{3, \mathrm{c}}$, Xiaocui Zheng ${ }^{4, \mathrm{~d}}$ and \\ Fei Wang ${ }^{5, e^{*}}$ \\ 1,4,5 Institute of security technology and engineering, Department Of Engineering Physics, Graduate \\ School at Shenzhen, Tsinghua University, Shenzhen 518055, China \\ ${ }^{2}$ Institute of Geomatics, Department of Civil Engineering, Tsinghua University, Beijing 100084, China \\ ${ }^{3}$ Tsinghua University State Key Lab of Hydro-Science and Engineering, Hydraulic Engineering \\ Department Room 310, New Hydraulic Building, Beijing 100084, China

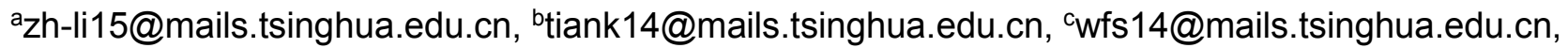 \\ dzheng.xiaocui@sz.tsinghua.edu.cn, eWang.fei@sz.tsinghua.edu.cn (Fei Wang ${ }^{*}$ is the \\ corresponding author)
}

Keywords: Remote Sensing, Damage Estimation, Crowdsourcing, Convolutional Neural Networks, Deep Learning, Image Processing

Abstract. Recently, natural disasters like earthquake, flooding, and landslides evoke our intensive awareness. The first priority to be concerned is the home status left by people, because from which can we largely estimate the damage level of the catastrophe and manage the rescue action better. However, to investigate the problem requires amount of expertizes working day and night, scrutinizing on the satellite images of the suffering areas and tagging damage levels to each building inefficiently. Thanks to both the ideas, the crowdsourcing approaches and widespread applications of machine learning (especially deep learning) dominating the science world recently, this issue can be easily tackled in a noble way. This paper presents our ideas of how to utilize our established platform to help simplify the problems with crowdsourcing ideas and Convolutional Neural Networks approaches to make effective automatic home damage level estimation.

\section{Introduction}

In the last two decades, we have witnessed the sprouting of complicated transducers and Big Data technology advances in calculating, transmitting and archiving a deluge of data. This revolutionary trend tide, which enables us to tackle problems from a more precise perspective by quantize the issue, is shaping our way of living [2, 4]. Crowdsourcing and Machine Learning, as mentioned in our paper, targets at fast and precisely analyzing huge data sets by reducing dimensions of the convoluted questions to delegate the tasks to a scattered web of human processing and by training the computers to help us recognize and analysis the problems, through which, finally free our human work [3]. For instance, when disaster occurs, a spate of spatial and satellite image datasets can be demarcated into tiny fractions and posted online for volunteers to tag the home status of the damages areas with the established criteria. And those annotated images could be used as the training sets for computers to start machine learning, and finally to analyzing them automatically [1].

Crowdsourcing ideas, recognizing as the "wisdom of the crowds", has been increasing its application in swarming fields [4]. It has the advantage of intelligence surmounting individual experts' contribution. A nice and delicate design of the crowdsourcing tasks can attract volunteers' eyes, and get them immersed into the games designed as the crowdsourcing tasks. Numerous examples of successful crowdsourcing projects have been applied and have approved an unimaginable achievements. Cases like Wikipedia, a platform for its users to add and revise items to its knowledge data base, is benefiting our lives every seconds, exceling the previous traditional encyclopedia in aspects of knowledge renewal and users' feedback and interaction. In the last a few decades, thanks to the development of widely spread connection and social networks, crowdsourcing applications is getting in fashion and mature. 
Image classification is one of the principle application of machine learning. However, traditional approaches are limited both in the area of accuracy and adaptability for classifying images to the right categories [3]. There are plenty of attempts to improve the performance, including collecting larger datasets, building more complicated and hodgepodge models, and using more powerful computers. But still, the detecting rate is unsatisfied due to the limitations of the traditional machine learning methods $[5,6]$.

In our house damage estimation case, we require a model with capable learning potential. Traditional machine learning approaches, however, could not meet the goals for the tremendous intricacy of the image classification tasks. Convolutional Neural Networks (CNNs), on the other side, enlightens this problem with the capacity of adapting its depth and breadth and with cogent understand about the nature of images [7]. The statistic stability and local pixel dependency of the Convolutional Neural Networks enable it be trained much more easier and perform better.

Our main contributions of this paper are as follows: we established a platform to get volunteers involved in crowdsourcing our projects, which will be introduced in section 2. Namely, we built interfaces for choosing the best satellite images from different providers, tagging chosen satellite images and uploading requirements and information from the disaster area. We also came up with the idea of applying Convolutional neural networks to train the tagged data, detailed in section 3, to make the estimation process automatically.

\section{Crowdsourcing Platform}

Platform for choosing the best satellite images from different providers

The quality of the satellite images of the disaster areas, if used for house status analysis, must have pretty well resolutions and very clear objects without clouds, mars, and deviations. In fact, those appropriate images are sometimes quite hard to obtain. Commercial remote sensing providers may offer services to meet our goals. The prohibitive cost and arduous procedures, however, avert the time effectiveness in fund and administration approving. Fortunately, Great IT corporations as Google, Microsoft, Baidu and Alibaba appropriate their own aerial databases and are open to public. Even though there are license problems, we can still use it for our research field.

As shown in Fig. 1, we adopted satellite images from Google map, MapBox, Amap (which is a Chinese provider named under Alibaba), and Esri. Volunteers can sign in our platform with their registration number and start contributing by choosing the best satellite images used for estimating the damage level of the houses from these satellite images providers.

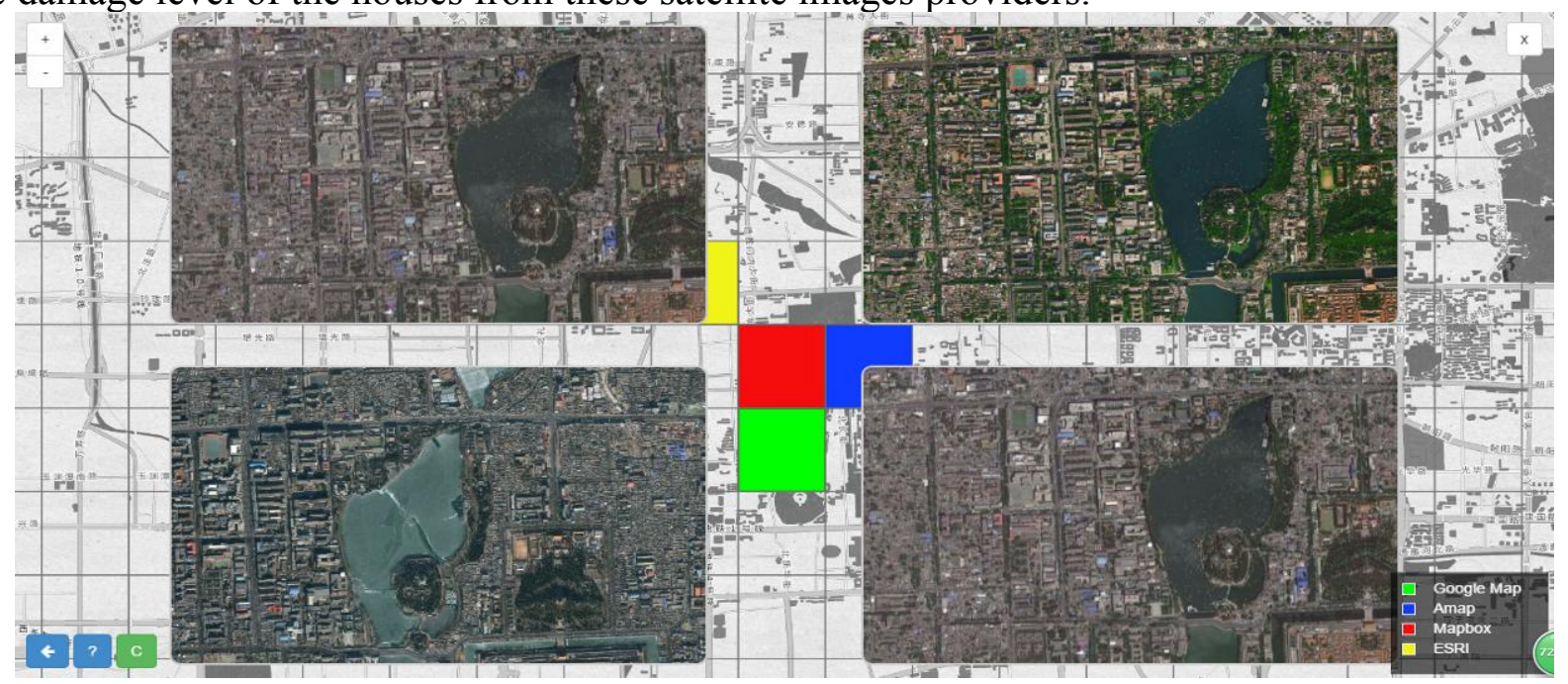

Fig. 1 Selected images mapping (satellite images are taken by Google Map, Amap, Mapbox and esri; the base map is rendered by GeoHey)

Platform for tagging the damage level

After choosing the best satellite images, our focus here is to estimate the damage level. From the aerial perspective, we encourage volunteers to judge the damage level from broader domain scale 
with the made criteria. Moreover, we also cherish them to upload the local building pictures taken in the site, and enable them to estimate the damage level of individual buildings from a local perspective with established standards.

\section{Mapping all the collected information on our maps}

Finally, we intend to show all the information on our emergency management maps. In these maps, we also add the statistic information given in the scale of the picture. Fig. 2 (rendered by GeoHey) illustrates the local mapping of the damage level of individual buildings..

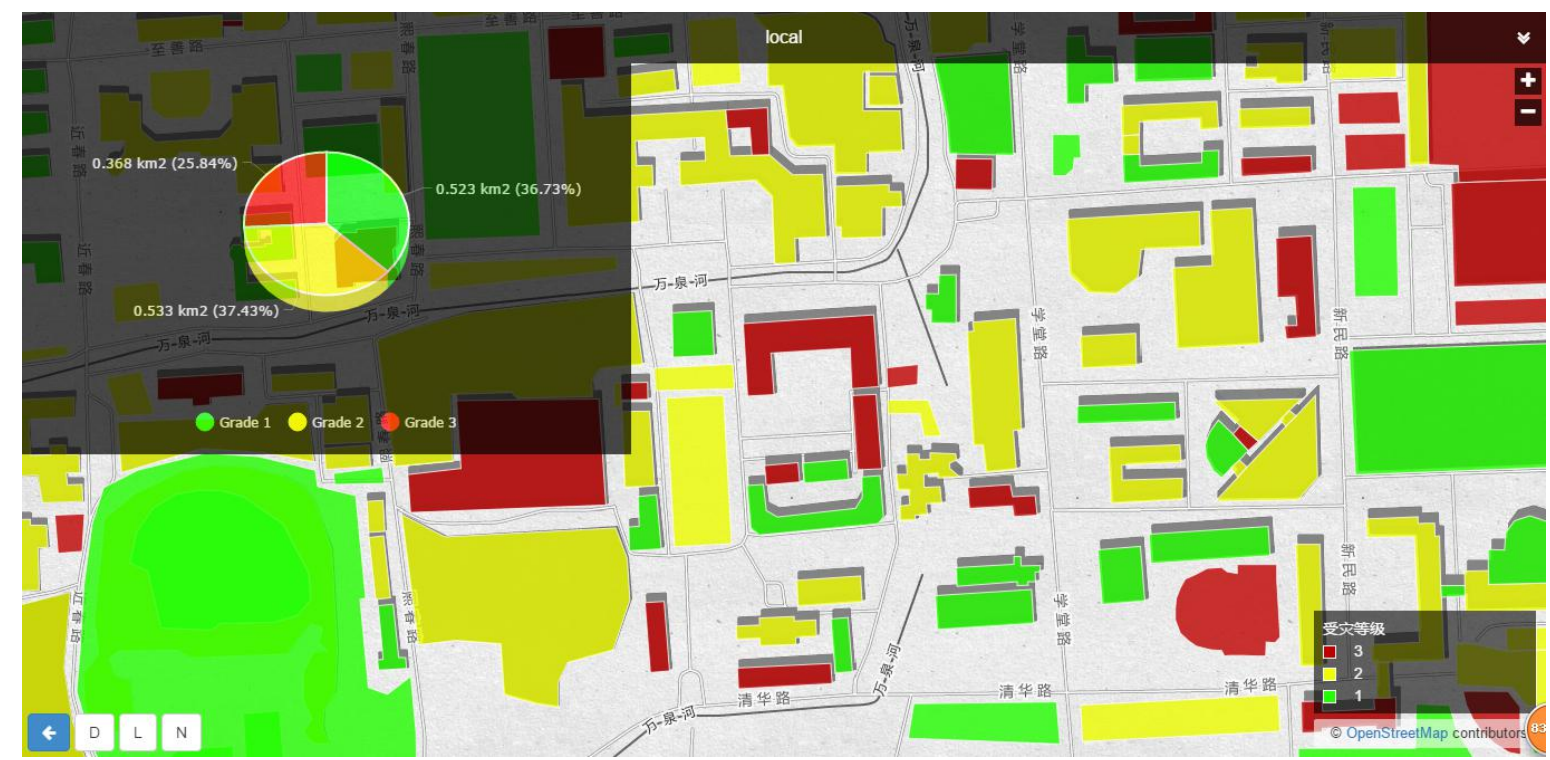

Fig. 2 Statistic mapping of the local damage level (the base map is rendered by GeoHey)

\section{Automatically estimating using Convolutional Neural Networks}

After having enough tagged data, we intend to make the estimation process automatically using Convolutional Neural Networks (CNNs). $\mathrm{CNN}$ is a multi-layered network. Each layer contains many two-dimension planes, and every plane embraces an avalanche of neural units. Some typical architecture of the Convolutional Neural Networks have several layers of networks with hidden layers [7]. The neural unites in planes of each layer are responsible for extracting a specific idiosyncrasy. The weight of the neural units in hidden layers are continuing modified while training.

As shown in Eq. 1, $a_{1}\left(v, k_{1-1}, k\right)$ and $b_{1}(k)$ stands for connection coefficients of positive and negative input respectively. $r_{l}(k)$, which is a constant, controls the eigenvalues selection. Higher the value is, the worse of the fault-tolerance for noise and distortion. But on another side, higher value of the $r_{l}(k)$ can be more likable to leads to a non-zero output, which betrays that this connection is of more significance [3].

$$
u_{s l}(k, n)=r_{l}(k) \phi\left[\frac{1+\sum_{k_{l-1}}^{K_{l-1}} \sum_{v \in A_{l}} a_{l}\left(v, k_{l-1}, k\right) u_{c l-1}\left(k_{l-1}, n+v\right)}{1+\frac{r_{l}(k)}{r_{l}(k)+1} b_{1}(k) u_{v l}(n)}-1\right]
$$

Convolutional Neural Networks is quite capable of managing record breaking consequences on our home damage level estimation project's dataset using supervised learning. A large quantity of datasets and extensive experiments are needed to be conducted to examine our work, which left for future work. 


\section{Conclusions}

This paper demonstrate our approaches and implementations of a crowdsourcing platform to estimate home damage levels in disaster areas. Through choosing the best satellite images from different source providers and asking volunteers to contribute crowd source damage estimation, our platform adopts collective wisdom to help tackle the arduous issues for specialists. After acquiring enough tagged datasets, we are able to use Convolutional Neural Networks to train our machines and foster them to substitute manual work in the future.

\section{Acknowledgements}

This work was funded by Technical Innovation Project of Shenzhen (CXZZ20140416155802785), Fundamental Research Project of Shenzhen (JCYJ20150331151358139), and Guangdong Emergency Platform Technology Research Center.

\section{References}

[1] Wang J, Zhang Z, Premachandran V, et al. Object Detectors Emerge in Deep Scene CNNs [J]. Computer Science, 2015.

[2] Chen L C, Barron J T, Papandreou G, et al. Semantic Image Segmentation with Task-Specific Edge Detection Using CNNs and a Discriminatively Trained Domain Transform [J]. Computer Science, 2015.

[3] Krizhevsky A, Sutskever I, Hinton G E. ImageNet Classification with Deep Convolutional Neural Networks [J]. Advances in Neural Information Processing Systems, 2012, 25(2):2012.

[4] Barrington L, Ghosh S, Greene M, et al. Crowdsourcing earthquake damage assessment using remote sensing imagery [J]. Annals of geophysics = Annali di geofisica, 2011, 54(6):680-687.

[5] Chen H L, Liu D Y, Yang B, et al. A new hybrid method based on local fisher discriminant analysis and support vector machines for hepatitis disease diagnosis [J]. Expert Systems with Applications an International Journal, 2011, 38(9):11796-11803.

[6] D. Lu, Q. Weng. A survey of image classification methods and techniques for improving classification performance [J]. International Journal of Remote Sensing, 2007, 28(5):823-870.

[7] K. Fukushima, “Neocognitron: A self-organizing neural-network model for a Mechanism of Pattern recognition unaffected by shift in position, Biol. Cybern., vol.36, pp.193 - 202, 1980.

[8] Nie Y, Yao L. Brief Introduction of the European Macroseismic Scale 1998[J]. Recent Developments in World Seismology, 2003. 